\title{
GENERALIZATIONS OF BANACH-HAUSDORFF LIMITS
}

\author{
RICHARD G. COOKE
}

In a recent paper $[1],{ }^{1} \mathrm{~W}$. F. Eberlein introduced the notion of Banach-Hausdorff limits. We employ throughout $m$ to denote the space of all real bounded sequences [2, pp. 11 and 34]. The BanachHausdorff limits are real-valued functionals $L(x)$, defined over $m$, which are Banach limits $[2$, p. 34], i.e., which satisfy the four conditions

(i) $L(a x+b y)=a L(x)+b L(y) \quad(a, b$ real $)$

(ii) $L(1)=1$,

(iii) $L(x) \geqq 0$ if $x \geqq 0$ (i.e., if $x_{n} \geqq 0$ for all $n$ ),

(iv) $L(S x)=L(x)$, where $S$ is the "translation" matrix $s_{n, n+1}=1$ $(n=1,2, \cdots), s_{n, k}=0(k \neq n+1)$, so that $S\left(x_{n}\right)=x_{n+1}$, and which, in addition to being Banach limits, satisfy the condition

(v) $L(H x)=L(x), H \in \mathcal{H C}_{+}$,

where $\mathcal{H}_{+}$is the semi-group of non-negative regular Hausdorff matrices, ${ }^{2}$ i.e., the set of $T$-matrices [3, pp. 64-65] given by

$$
h_{n, k}={ }_{n} C_{k} \int_{0}^{1} u^{k}(1-u)^{n-k} d \alpha(u)(k \leqq n), h_{n, k}=0(k>n),
$$

$\alpha(u)$ being a nondecreasing function of $u$, with $\alpha(0)=\alpha(+0)=0$, $\alpha(1)=1$ (so that $h_{n, k} \geqq 0$ for all $n$ and $k$ ).

Writing $L^{*}(x)=\lim \sup _{n \rightarrow \infty} x_{n}, L_{*}(x)=\lim _{i n f} x_{n \rightarrow \infty} x_{n}$, and

$$
P_{+}(x)=\inf _{H \in \mathcal{C}_{+}} L^{*}(H x), \quad P_{-}(x)=-P_{+}(-x)=\sup _{H \in \mathcal{J}_{+}} L_{*}(H x),
$$

where $x \in m$, Eberlein proves the following theorem:

$(\alpha)^{\prime} A$ necessary and sufficient condition that the linear functional $L(x)$ on $m$ should be a Banach-Hausdorff $(B-H)$ functional is that

$$
P_{-}(x) \leqq L(x) \leqq P_{+}(x) \quad(x \in m) ;
$$

$(\beta)^{\prime} B$-H functionals exist;

$(\gamma)^{\prime} A$ necessary and sufficient condition that all $B-H$ functionals coincide at $x$ is that $P_{-}(x)=P_{+}(x)$.

This theorem is generalized in the present note in two distinct ways: (A) by replacing $\mathfrak{H C}_{+}$by other semi-groups of $T$-matrices, and

Received by the editors April 29, 1952, and, in revised form, October 14, 1952.

1 Numbers in brackets refer to the references at the end of the paper.

2 Eberlein calls such Hausdorff matrices completely regular. 
(B) by replacing the Banach limits by a wider class of generalized limits recently introduced by R. E. Edwards [4], in which the translative condition $L(S x)=L(x)$ of Banach is not necessarily satisfied. ${ }^{3}$

We consider first the generalization (A).

To replace $\mathcal{F}_{+}$, we consider a semi-group $\mathcal{A}$ of non-negative $T$ matrices with the following properties: (a) every pair of matrices $A, B$ of $A$ is such that $A B$ and $B A$ are absolutely equivalent for $m[3$, pp. 97 and $105,(5.4, \mathrm{I})],{ }^{4}$ (b) $\sum_{k=1}^{\infty} a_{n, k}=\sum_{k=1}^{\infty}\left|a_{n, k}\right|=1$ for every $n$, and (c) $\mathcal{A}$ contains at least one matrix which is absolutely regular (absolutely translative) for $m[3, \mathrm{pp} .114,119]$.

We first make some remarks on the above properties of $\mathcal{A}$. If $A$ and $B$ commute, then (a) is satisfied; thus (a) is satisfied by $\mathfrak{C}_{+}$. But the converse is false, so that (a) is less restrictive than commutability; it implies that the $A$ and $B$ limits are consistent for $m[3$, p. 132, Ex. 11].

The matrix used by Eberlein which satisfies (c) is that of the arithmetic means $H_{0}$ [see 3, p. 120 (5.6, III), and p. 132, Ex. 10]; this is contained in $A$ when $A$ is $\mathfrak{H C}_{+}$.

Every matrix $A$ of $A$ is a stochastic matrix [5], a type employed in statistics; in addition to the class $\mathfrak{H C}_{+}$, examples of these are the Borel matrix [3, pp. 69-70], the Abel $T$-matrix [3, p. 218], the Nörlund matrix $[3$, p. 73$]$, the Taylor series continuation matrix $F(p)(0<p$ $\leqq 1)[6$, p. $548,(3.16)]$, and the Laurent series continuation matrix $[6$, p. $558,(5.8)]$.

To show that $A$ is a semi-group, we note that the product of any number of non-negative $T$-matrices is a non-negative $T$-matrix [3, p. 83, (4.6, II)], and that if $A, B, C$ are in $A$, then each of the pairs $(C A B, C B A)$ and $(B C A, C B A)$ are absolutely equivalent for $m$ [see 3 , p. 133, Ex. 15], and hence the same is true of the pair $(C A B, B C A)$; i.e., products of matrices of $A$ have the property (a). Also since $\|A\|$ $=1$, and $\|B\|=1$, where $\|A\|$ is the $K_{r}$-bound of $A[3$, pp. 25-26] $(A, B \in \mathcal{A})$, we have $\|A B\| \leqq\|A\|\|B\|=1$, so that $\|A B\|=1$, since $\|A B\|$ cannot be less than 1 .

We term $L(x)$ a $B$-eA functional or limit on $m$ if, in addition to being a Banach limit, it satisfies the condition

(vi) $L(A x)=L(x), A \in \mathcal{A}, x \in m$;

\footnotetext{
3 It has been pointed out to me by Dr. G. G. Lorentz and Dr. Y. N. Dowker that these generalized limits of Edwards had been previously given (in a more general form) by R. Sikorski, Studia Math. vol. 12 (1951) pp. 117-124.

- This theorem is actually proved for $\sigma_{\infty}$, the space of all complex bounded sequences; but it will be seen from the proof that when the matrices concerned are real (as in the present case), $\sigma_{\infty}$ may be replaced by $m$.
} 
thus if $x \in m$ is summable to $s$ by some matrix $A \in \mathcal{A}$, then $L(x)=s$ for every $B \cdot \mathcal{A}$ functional $L$.

With the meanings for $L^{*}(x)$ and $L_{*}(x)$ quoted above, we then define $P_{+}(x)=\inf _{A \in \mathcal{C}} L^{*}(A x), P_{-}(x)=-P_{+}(-x)=\sup _{A} \in \mathcal{A} L_{*}(A x)$; the following result can then be established along lines similar to the proof of Eberlein's theorem.

Theorem. ( $\alpha$ ) A necessary and sufficient condition that the linear functional $L(x)$ on $m$ should be a $B-\mathcal{A}$ functional is that

$$
P_{-}(x) \leqq L(x) \leqq P_{+}(x) \quad(x \in m) ;
$$

( $\beta$ ) $B-A$ functionals exist;

( $\gamma$ ) $A$ necessary and sufficient condition that all $B-A$ functionals coincide at $x$ is that $P_{-}(x)=P_{+}(x)$.

The notation $x \sim y$ means that $x-y$ is a null sequence, i.e., $x_{n}$ $-y_{n} \rightarrow 0$ as $n \rightarrow \infty$.

Lemma 1. By property (c) of $\mathcal{A}$, there exists a matrix $A^{(0)}$ of $\mathcal{A}$ which is absolutely regular (absolutely translative) for all $x$ in $m$; we then have

$$
A^{(0)} S x \sim A^{(0)} x \quad \text { for all } x \in m .
$$

For, $\quad\left(A^{(0)} S x-A^{(0)} x\right)_{n}=-a_{n, 1}^{(0)} x_{1}+\sum_{k=1}^{\infty}\left(a_{n, \mathrm{k}}^{(0)}-a_{n, \mathrm{k}+1}^{(0)}\right) x_{k+1} \rightarrow 0 \quad$ as $n \rightarrow \infty$ for all $x \in m$ if, and only if,

$$
\sum_{k=1}^{\infty}\left|a_{n, k}^{(0)}-a_{n, k+1}^{(0)}\right| \rightarrow 0 \quad \text { as } n \rightarrow \infty,
$$

which is true, by hypothesis [3, p. 119, $(5.6, I V)]$.

It appears unnecessary to show also that $A^{(0)} x \sim S A^{(0)} x$, in analogy to Eberlein's $H_{0} x \sim S H_{0} x$, since Eberlein does not use the latter anywhere in his proof; it would require the extra condition

$$
\sum_{k=1}^{\infty}\left|a_{n, k}^{(0)}-a_{n+1, k}^{(0)}\right| \rightarrow 0 \quad \text { as } n \rightarrow \infty .
$$

LEMma 2. (a) $P_{+}(x+y) \leqq P_{+}(x)+P_{+}(y) ;(\mathrm{b})^{\prime} \quad P_{+}(a x)=a P_{+}(x)$, $a \geqq 0$; (c) $)^{\prime} P_{-}(x) \leqq P_{+}(x)$; (d)' $\left|P_{ \pm}(x)\right| \leqq\|x\|=\sup _{n}\left|x_{n}\right| ; P_{ \pm}(x) \geqq 0$ if $x \geqq 0$; (e) ${ }^{\prime}$ if $\left\{x_{n}\right\}$ is convergent, then $P_{ \pm}(x)=\lim _{n \rightarrow \infty} x_{n}$, and $P_{ \pm}(x+y)=\lim _{n \rightarrow \infty} x_{n}+P_{ \pm}(y)$; in particular, $P_{ \pm}(x)=P_{ \pm}(y)$ if $x \sim y$; (f) ${ }^{\prime} P_{ \pm}(A x)=P_{ \pm}(x)(A \in \mathcal{A}) ;(\mathrm{g})^{\prime} P_{ \pm}(S x)=P_{ \pm}(x) ;(\mathrm{h})^{\prime} P_{ \pm}(S x-x)=0$; (i) $P_{ \pm}(A x-x)=0(A \in \mathcal{A})$.

We need only comment on (a) ${ }^{\prime}$ and (i)'; the proofs of the others are exactly as given by Eberlein. 
(a)'. Given $\epsilon>0$, there exist $A^{(1)}$ and $A^{(2)}$ in $A$ such that $L^{*}\left(A^{(1)} x\right)$ $\leqq P_{+}(x)+\epsilon, L^{*}\left(A^{(2)} y\right) \leqq P_{+}(y)+\epsilon$. Now $L^{*}\left(A^{(1)} A^{(2)} x-A^{(2)} A^{(1)} x\right)=0$, since $A^{(1)} A^{(2)}$ and $A^{(2)} A^{(1)}$ are absolutely equivalent for $m$ by property (a) of $A$, so that $A^{(1)} A^{(2)} x-A^{(2)} A^{(1)} x \sim 0$. Also $L^{*}\left(A^{(1)} A^{(2)} x\right)$ $\leqq L^{*}\left(A^{(2)} x\right)$, by Knopp's core theorem $[3$, p. $138,(6.1,1)]$. Hence, writing $A^{(3)}=A^{(1)} A^{(2)}$, we have

$$
\begin{aligned}
L^{*}\left(A^{(3)} x\right) & =L^{*}\left(A^{(1)} A^{(2)} x\right) \\
& \leqq L^{*}\left(A^{(1)} A^{(2)} x-A^{(2)} A^{(1)} x\right)+L^{*}\left(A^{(2)} A^{(1)} x\right) \\
& =L^{*}\left(A^{(2)} A^{(1)} x\right) \leqq L^{*}\left(A^{(1)} x\right) \leqq P_{+}(x)+\epsilon,
\end{aligned}
$$

and

$$
L^{*}\left(A^{(3)} y\right)=L^{*}\left(A^{(1)} A^{(2)} y\right) \leqq L^{*}\left(A^{(2)} y\right) \leqq P_{+}(y)+\epsilon .
$$

Thus $P_{+}(x+y) \leqq L^{*}\left\{A^{(3)}(x+y)\right\} \leqq L^{*}\left(A^{(3)} x\right)+L^{*}\left(A^{(3)} y\right) \leqq P_{+}(x)$ $+P_{+}(y)+2 \epsilon$, so that (a)' follows, $\epsilon$ being arbitrary.

(i) ${ }^{\prime}$ In proving the analogue of $(i)^{\prime}$ for $\mathfrak{H}_{+}$, Eberlein uses an ergodic property which he had previously established. This property extends easily to the present case.

For, since $\|A\|=1$ for every $A \in \mathcal{A}$, then, if $A_{n}=(1 / n) \sum_{k=1}^{n} A^{k}$, where $A \in \mathcal{C}$, it is obvious that $\left\|A_{n}\right\|=1$ and that $A_{n} \in \mathcal{A}$. Also, $A_{n} A-A_{n}=(1 / n) \sum_{k=1}^{n}\left(A^{k+1}-A^{k}\right)=(1 / n)\left(A^{n+1}-A\right)$, so that $\| A_{n} A$ $-A_{n} \| \leqq(1 / n)\left(\|A\|^{n+1}+\|A\|\right)=2 / n$, since $\|A\|=1$. Hence by (f) ${ }^{\prime}$ and $(\mathrm{d})^{\prime}$,

$\left|P_{ \pm}(A x-x)\right|=\left|P_{ \pm}\left\{\left(A_{n} A-A_{n}\right) x\right\}\right| \leqq\left\|\left(A_{n} A-A_{n}\right) x\right\| \leqq 2\|x\| / n$.

Since $n$ is an arbitrary positive integer, this shows that $P_{ \pm}(A x-x)$ $=0$, so that $(i)^{\prime}$ is established.

PROOF OF THE THEOREM. This follows exactly as in the parallel proof of Eberlein, merely substituting " $B-\mathcal{C} A$ functional" for " $B-H$ functional."

COROLLARY. The theorem remains true if, in the definition of the semigroup $A$, condition (b) is replaced by the following more general condition (b)': Every matrix $A \in \mathcal{C} A$ is of the form $A=B+C$, where $B$ satisfies condition (b), and $C$ transforms every bounded sequence into a null sequence (i.e., $A$ and $B$ are absolutely equivalent for all bounded sequences).

Let $M_{n}=\sum_{k=1}^{\infty} a_{n, k}=\sum_{k=1}^{\infty}\left|a_{n, k}\right|$, and write $\|A\|=\sup _{n} M_{n}$, the $K_{r}$-bound of $A$.

If $M_{n} \leqq 1$ for every $n$, then $\|A\|=1$. If $M_{n}>1$ for one or more values of $n$, then $\|A\|>1$; in this case we write $b_{n, k}=a_{n, k} / M_{n}$, so that $B$ satisfies condition (b), and consequently $\|B\|=1$. Also 


$$
\sum_{k=1}^{\infty}\left|a_{n, k}-b_{n, k}\right|=\sum_{k=1}^{\infty}\left(a_{n, k}-b_{n, k}\right)=M_{n}-1 \rightarrow 0 \quad \text { as } n \rightarrow \infty,
$$

so that $A$ and $B$ are absolutely equivalent for $m$. Thus $A$ satisfies condition (b)'.

It also follows that condition (a) in the definition of $A$ is satisfied by $B$, for, since $A$ and $B$ are absolutely equivalent for $m$, the same is true of $A B$ and $B A[3,133$, Ex. 12].

It remains only to show that (i) $)^{\prime}$ of Lemma 2 holds in the case when (b) ${ }^{\prime}$ is assumed in the place of (b).

If $\|A\|=1$, the proof already given applies. If $\|A\|>1$ for some $A \in \mathcal{C} A$, we obtain, as before, $\left|P_{ \pm}(B x-x)\right| \leqq 2\|x\| / n$, where $b_{n, k}$ $=a_{n, k} / M_{n}$, so that $\|B\|=1$. Thus $P_{ \pm}(B x-x)=0$. But, by (e) of Lemma $2, P_{ \pm}(B x-x)=P_{ \pm}(A x-x)$, since $A$ and $B$ are absolutely equivalent for $m$, so that $B x-x \sim A x-x$.

The corollary now follows.

If $A$ contains $S$, the translation matrix, the proof is simplified, since (iv), i.e., $L(S x)=L(x)$, follows immediately as a particular case of (vi), and (g)' and (h) of Lemma 2 are then no longer required. If $S \in \mathcal{A}$, property (a) of $\mathcal{A}$ requires that $\sum_{k=1}^{\infty}\left|a_{n, k-1}-a_{n+1, k}\right| \rightarrow 0$ as $n \rightarrow \infty$ for every $A \in \mathcal{C}$, which places rather a heavy restriction on $\mathcal{A}$.

Of course $\mathcal{F}_{+}$cannot contain $S$, since $S$ is not a lower semi-matrix (apart from other considerations).

We now consider the generalization (B).

The generalized limits of Edwards, mentioned above, do not always satisfy the restrictive translation condition (iv) of Banach. These limits appear in the representation by Edwards [4] of the most general continuous linear functional on certain spaces of functions, of which $m$ is a particular example, the representation involving both generalized limits and Radon measures.

Edwards defines a generalized limit on $m$ as a linear functional $L(x)$ satisfying

(vii) $L(x) \leqq L^{*}(x), x \in m$;

thus condition (i) of Banach is assumed.

Then $L(-x)=-L(x)$, and so (vii) gives $-L(x) \leqq L^{*}(-x)$, i.e., $L(x) \geqq L_{*}(x)$; hence $L(x) \geqq 0$ if $x \geqq 0$, so that (iii) is satisfied. Also $L_{*}(1)=L^{*}(1)=1$, so that $L(1)=1$, i.e., (ii) is satisfied. But (iv) need not be satisfied.

Now if $L(x)$ is defined by (vii), and the definition of $A$ remains as before (so that property (c) is still assumed), then the above theorem and its corollary hold without any modification, so that (iv) holds; 
in this case the $L(x)$ of the theorem consist of a subclass of Edwards' generalized limits which are also Banach limits, and they are, of course, $B-A$ functionals.

Consider, however, a new semi-group $\mathcal{A}^{\prime}$, in which the properties (a) and (b) or $\left(\mathrm{b}^{\prime}\right)$ of $\mathcal{A}$ are retained, but (c) is replaced by (d): $\mathcal{A}^{\prime}$ contains no matrix which is absolutely regular (absolutely translative) for $m$.

Then for $\mathcal{A}^{\prime}$, Lemma 1 of the above theorem is false, and so must be omitted; and consequently conditions $(\mathrm{g})^{\prime}$ and $(\mathrm{h})^{\prime}$ of Lemma 2 must be omitted. Condition (iv) of Banach need not then be satisfied by $L(x)$.

We call $L(x) a B^{*}-A^{\prime}$ functional or limit if it satisfies (vii) and the condition

(viii) $L(A x)=L(x), A \in A^{\prime}, x \in m$.

We also define

$$
P_{+}^{\prime}(x)=\inf _{\Delta \in \mathcal{C} A^{\prime}} L^{*}(A x), \quad P_{-}^{\prime}(x)=-P_{+}^{\prime}(-x)=\sup _{A \in \mathcal{C}} L^{\prime} L_{*}(A x),
$$

and then obtain the following result.

Theorem. ( $\delta$ ) $A$ necessary and sufficient condition that the linear functional $L(x)$ defined by (vii) should be a $B^{*}-A^{\prime}$ functional is that

$$
P_{-}^{\prime}(x) \leqq L(x) \leqq P_{+}^{\prime}(x) \quad(x \in m) ;
$$

(є) $\left.B^{*}\right\lrcorner A^{\prime}$ functionals exist;

( $\eta$ ) $A$ necessary and sufficient condition that all $B^{*}-A^{\prime}$ functionals coincide at $x$ is that

$$
P_{-}^{\prime}(x)=P_{+}^{\prime}(x) .
$$

It is only necessary to prove that $B^{*}-A^{\prime}$ functionals exist. If, for any $T$-matrix $A, p_{n}$ is defined as the smallest integer for which $\left|a_{n, p_{n}}\right|=\max _{k=1,2}, \ldots\left|a_{n, k}\right|$, and if

$$
\sum_{k=1}^{\infty}\left|a_{n, k}-a_{n, k+1}\right| \rightarrow 0 \quad \text { as } n \rightarrow \infty,
$$

then

$$
\sum_{k=1}^{p_{n}-1}\left|a_{n, k}-a_{n, k+1}\right| \rightarrow 0, \quad \text { and so } \quad \sum_{k=1}^{p_{n}-1}\left(a_{n, k}-a_{n, k+1}\right) \rightarrow 0,
$$

i.e., $a_{n, 1}-a_{n, p_{n}} \rightarrow 0$. Since $A$ is a $T$-matrix, it follows that

$$
a_{n, p_{n}} \rightarrow 0
$$

as $n \rightarrow \infty$. 
Thus ( $\mathrm{x}$ ) follows from (ix), but the converse is false. Now (x) is a sufficient condition that $A$ should sum some divergent sequences of 0 's and 1's."

Hence we require $A^{\prime}$ to consist of matrices $A$ (with properties (a) and (b) or (b)' of $\mathcal{A}$ ) for some, at least, of which (x) holds, but for none of which (ix) holds.

Such matrices may be obtained in the following way.

Take the matrices of $\mathcal{A}$, and form corresponding "diluted" and "row-repeated" matrices by inserting one or more columns of zero elements between every consecutive pair of columns of each matrix of $\mathcal{A}$, the insertions being the same for each such matrix, and by repeating the $n$th row $(n=1,2, \cdots)$ of each such matrix $m_{n}$ times, where $m_{n}$ is the number of zero columns inserted between the $n$th and $(n+1)$ th columns of the original matrix of $A$. Thus if only one column of zeros is inserted between each column of $A \in \mathcal{C} A$ becomes

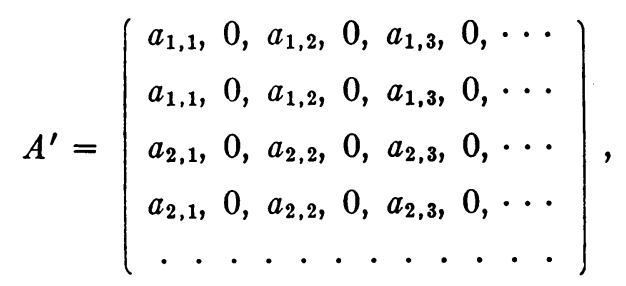

where each row of $A$ is repeated once.

Then $A$ thus modified forms a suitable $A^{\prime}$. For, property (b) or (b)' of $\mathcal{A}$ is still satisfied by the non-negative $T$-matrices $A^{\prime}$ of $\mathcal{A}^{\prime}$, and property (a) of $\mathcal{A}$ is also satisfied, since the latter gives

$$
\sum_{k=1}^{\infty}\left|\sum_{i=1}^{\infty}\left(a_{n, i} b_{i, k}-b_{n, i} a_{i, k}\right)\right| \rightarrow 0 \quad \text { as } n \rightarrow \infty,
$$

and

$$
\sum_{k=1}^{\infty}\left|\sum_{i=1}^{\infty}\left(a_{n, i}^{\prime} b_{i, k}^{\prime}-b_{n, i}^{\prime} a_{i, k}^{\prime}\right)\right| \quad\left(A^{\prime}, B^{\prime} \in \mathcal{A}^{\prime}\right)
$$

consists of some of the terms of the series (xi), and hence, a fortiori, tends to 0 as $n \rightarrow \infty$.

4 This was proved by Agnew [see 3, pp. 204-205], and Dr. G. G. Lorentz informs me that he also gave it in the Bericht Tagung Tübingen, 1946, having previously submitted a paper containing a more general theorem in October 1943 to the Math. Zeit. (where it did not appear).

- The idea of using "diluted" matrices for this purpose was suggested to me by Dr. P. Vermes. 
By supposition, there is a matrix, say $A$, of $A$ which satisfies (ix), i.e. property (c) of $A$, and hence satisfies (x). This matrix $A$, when diluted and row-repeated as above, will still satisfy $(x)$, but will not satisfy (ix). Thus the existence of a nonempty $\mathcal{A}^{\prime}$ is established, and hence $B^{*}-A^{\prime}$ functionals exist.

Since the number of zero columns inserted in the matrices of $\mathcal{A}$ is arbitrary, it is evident that $B^{*}-A^{\prime}$ functionals are much more numerous than $B-A$ functionals.

I have pleasure in thanking Dr. P. Vermes for some useful comments on the manuscript, and Dr. R. E. Edwards for some remarks.

\section{REFERENCES}

1. W. F. Eberlein, Banach-Hausdorff limits, Proceedings of the American Mathematical Society vol. 1 (1950) pp. 662-665.

2. S. Banach, Theorie des operations linéaires, Warsaw, 1932.

3. R. G. Cooke, Infinite matrices and sequence spaces, Macmillan, 1950.

4. R. E. Edwards, On the weak convergence of continuous functions, Neder. Akad. Wetensch. Ser. A, vol. 55 (1952) pp. 230-236.

5. T. A. Sarymsakov, On sequences of stochastic matrices, C.R. (Doklady) Acad. Sci. URSS. N.S. vol. 47 (1945) pp. 326-328.

6. P. Vermes, Series to series transformations and analytic continuation by matrix methods, Amer. J. Math. vol. 71 (1949) pp. 541-562.

Birkbeck College, University of London 